International Review of Research in Open and Distributed Learning Volume 18, Number 3

May - 2017

\title{
Book Review: Conducting Qualitative Research of Learning in Online Spaces
}

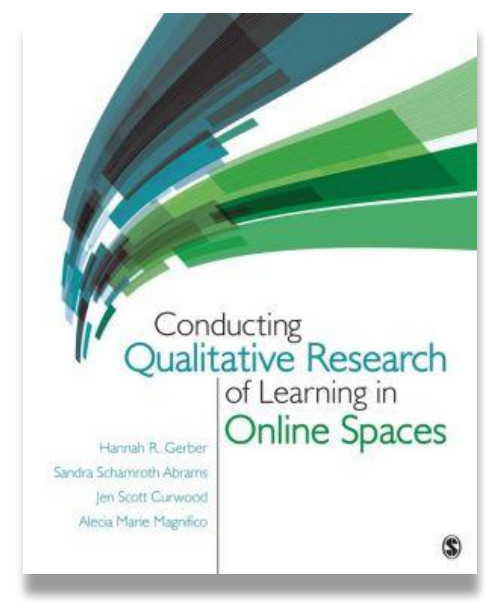

Written By: Hannah R. Gerber, Sandra S. Abrams, Jen S. Curwood, and Alecia M. Magnifico (Thousand Oaks, CA: SAGE Publications Inc, 2016, 232 pages)

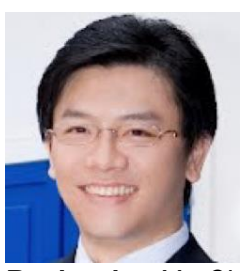

Review by: Yu-Chang Hsu, PhD, Department of Educational Technology, Boise State University

I reviewed this book from the perspective of a researcher/scholar of online learning, a teacher of research methodology, and a colleague of academia, which should reflect in my review in reciprocal fashion.

This book, while not extensive, is well-structured and well-written. The authors effectively and efficiently deliver on the various aspects of conducting qualitative research of learning in online spaces. The combined rich and varied experiences from the authors in qualitative research experiences are impressive. Readers of this book can benefit from the consistent structure and concise language used in explaining important aspects on conducting qualitative research in online spaces. The authors succinctly yet clearly discuss research paradigms and philosophical stances early in the book. This provides a good basis for their book. It will help inform different level of graduate students and serve as a refresher for seasoned researchers.

This book also strikes a good balance between discussions of research methods and those of research contexts (i.e., online spaces). While online spaces vary to a great extent, the authors present concise summaries of characteristics for each type of space and provide examples of research efforts to make the case for their discussions. There are appropriate references and discussions of classical works related to different methods. More recent research projects are also cited and discussed, reflecting the focus of different methods, data collection, and data analysis. There is a total of 16 figures (e.g., screenshots of online field sites), 10 tables (e.g., categories and examples of online spaces for learning), and 17 Spotlight Boxes. While the figures and tables can be a great resources for graduate students and research course instructors alike, the topical summaries provided in Spotlight Boxes (e.g., Capturing Dynamic Learning with Stimulated Recall, or Multimodal Microanalysis) can be invaluable windows to research projects related to specific topics of focus (i.e., data collection and data analysis) in the book. Each of the eight chapters start with Guiding Questions for readers to warm up for the learning journey, and end with Connecting to Your 
Work to extend the learning/thinking. The four authors present a consistent voice, and the prose is clear and concise. The forward by Onwuegbuzie is also worth visiting. It reaffirms the timeliness and necessity of this book, and contextualizes it in an era of increasingly more and diverse online spaces of learning.

While this is a great method book related to researching online spaces, it would work better as a second book accompanying a foundational and comprehensive research method textbook for an introductory graduate course. Novice researchers might find the need to establish foundations and familiarity in overall research methodology, as well as terminology, before navigating this book. It could also serve as a major textbook in a more advanced qualitative method course. Experienced researchers will find the text flows well and the authors are well-versed in both worlds of research methods and online learning spaces.

\section{Athabasca University}

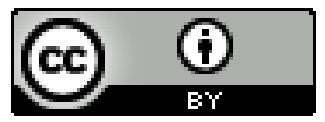

